\title{
Cascade Control of the Toycopter
}

\author{
Ph. Mullhaupt, B. Srinivasan, J. Lévine †, D. Bonvin \\ Institut d'automatique, Ecole Polytechnique Fédérale de Lausanne \\ CH-1015 Lausanne. \\ $\dagger$ Centre Automatique et Systèmes, \\ Ecole Nationale Supérieure des Mines de Paris \\ Fontainebleau. \\ e-mail: name@ia.epfl.ch, †levine@cas.ensmp.fr
}

\begin{abstract}
This paper deals with the control of a two-degreeof-freedom, laboratory-scale helicopter-like system (termed the toycopter), where the aerodynamic force is manipulated using the propeller speed. This system can be shown not to be flat and thus classical linearization techniques cannot be applied. However, a low-order flat system can be obtained by (i) using a high-gain feedback with suitable proportional gains on the propeller speeds, and (ii) neglecting the inertial cross coupling terms. The flatness can be exploited to ensure precise tracking of reference trajectories and good disturbance rejection.
\end{abstract}

\section{Introduction}

The design of controllers for highly nonlinear systems often calls for methods based on first-order linear approximations. The performance can typically be enhanced with the use of several linear approximations of the nonlinear system, using for example either multimodel linear regulator synthesis or gain scheduling $[2,5,7,12,14,15]$. Following the same idea of exploiting the best prior knowledge given by the original dynamics, it would be interesting to use nonlinear approximations rather than linear ones. It is the authors' opinion that flat systems are good candidates for such approximations since motion planning and stabilization can be handled in an elegant and straightforward manner despite their inherent nonlinear dynamics $[3,4]$. Variants of this idea are found in $[1,6,8,9]$.

This paper considers the case study of a twodegree-of-freedom, laboratory-scale helicopter-like system (termed the toycopter), where the aerodynamic force is manipulated using the propeller speed. Such a system is nonlinear with strong couplings and is not flat [11] [13]. This is due to the presence of cross-coupling stemming from the way the aerodynamical force is varied to control the system.
The system would be flat if the inputs were the propeller speeds and if the terms proportional to the propeller acceleration were absent. Thus, in order to meet approximatly these requirements, high-gain feedback loops are used so that the inputs control the propeller speeds. In addition, when the terms containing the propeller accelerations are neglected, the reduced-order system is flat. Based on this flat approximation, a cascade controller is designed that gives excellent trajectory tracking and good disturbance rejection.

The paper presents a three-level cascade structure to handle the control of the toycopter. The inner level, which consists in high-gain controllers, is followed by a linearizing controller and finally by outer controllers that stabilize the system around the reference trajectories.

The flat approximation is valid as long as the trajectories do not evolve too rapidly between the initial and final positions. The dominant time constant depends on the outer feedback loops. If the corresponding gains are not too large, The speed of evolution of the system depends on the external feedback. If the gains of this feedback are not too large the method based on the flat approximation is applicable.

Section 2 presents the setup and the model equations used throughout the paper. The flatness analysis is given in Section 3.1. A flat reduced-order model is given in Section 3.2. Section 4 presents the three-level cascade control scheme. Some of the limitations introduced by the approximation used to obtain the cascade structure are given in Section 5. Real-time experiments and comparison with simulation are considered in Section 6 , and Section 7 concludes the paper.

\section{Model of the Toycopter}

The setup under study is a rigid body mechanical system composed of two main links. The first link is positioned vertically and is articulated to the base through 


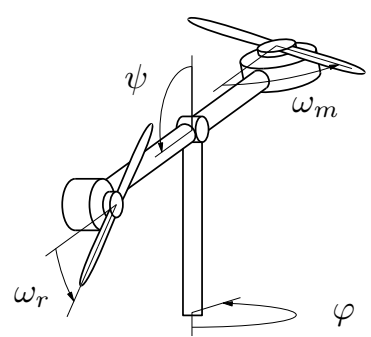

Figure 1: Helicopter model

a rotational joint, giving rise to the horizontal movement of the toycopter ( $\varphi$ coordinate). A second link, termed the arm, is articulated to the first link through another rotational joint permitting vertical movement ( $\psi$ coordinate). At both ends of the arm are mounted DC-motors, each equipped with a propeller. These motors are mounted such that their axis of rotation points in the direction of the movement they are actuating. The main motor varies its speed $\omega_{m}$ in order to control the aerodynamical force generated along the $\psi$ coordinate, the rear motor varies its speed $\omega_{r}$ to control the horizontal movement. Notice that, despite the fact that there are as many actuators as desired direction of movement, the system is nevertheless underactuated, since more generalized coordinates than independent actuators are present. This can be understood intuitively by saying that the motors have to control both their speed and the coordinate corresponding to the direction they are actuating. The center of mass of the system is purposely not on the articulation between the first and the second link, making the system unstable. The system has 6 states $\left\{\psi, \dot{\psi}, \varphi, \dot{\varphi}, \omega_{m}, \omega_{r}\right\}$ and two inputs, the voltages $\left\{u_{m}, u_{r}\right\}$ applied to the motors. The system equations read:

$$
\begin{aligned}
I_{\psi} \ddot{\psi}+ & I_{r} \dot{\omega}_{r}=C_{m} \omega_{m}\left|\omega_{m}\right|-C_{r 1} \omega_{r}\left|\omega_{r}\right|+ \\
& G_{s} \sin \psi+G_{c} \cos \psi+\frac{1}{2} I_{c} \dot{\varphi}^{2} \sin (2 \psi)- \\
& C_{\psi} \dot{\psi}+I_{m} \omega_{m} \dot{\varphi} \cos \psi
\end{aligned}
$$

$$
\begin{aligned}
\left(I_{\varphi}+I_{c} \sin ^{2}(\psi)\right) \ddot{\varphi}+ & I_{m} \dot{\omega}_{m} \sin \psi \\
= & C_{r} \omega_{r}\left|\omega_{r}\right| \sin \psi- \\
& C_{m 1} \omega_{m}\left|\omega_{m}\right| \sin \psi- \\
& I_{c} \dot{\psi} \dot{\varphi} \sin (2 \psi)-I_{m} \omega_{m} \dot{\psi} \cos \psi- \\
& C_{\varphi} \dot{\varphi}-C_{\varphi 0} \operatorname{sgn}(\dot{\varphi}) \\
I_{m} \dot{\omega}_{m}= & K_{m} u_{m}-F_{m} \omega_{m}-C_{m 1} \omega_{m}\left|\omega_{m}\right|
\end{aligned}
$$$$
I_{r} \dot{\omega}_{r}=K_{r} u_{r}-F_{r} \omega_{r}-C_{r 1} \omega_{r}\left|\omega_{r}\right|
$$

The main terms in the model are discussed next.
- Aerodynamical effects. The propellers generate torques that are proportional to the square of rotation speed. Alongwith the main driving torques $\left(C_{m} \omega_{m}\left|\omega_{m}\right|\right.$ and $\left.C_{r} \omega_{r}\left|\omega_{r}\right|\right)$, the propellers generate aerodynamical coupling due mainly to air resistance $\left(C_{m 1} \omega_{m}\left|\omega_{m}\right|\right.$ and $\left.C_{r 1} \omega_{r}\left|\omega_{r}\right|\right)$. The model given here differs from that in [11] where the aerodynamical forces were considered proportional to the propeller speeds.

- Inertial counter torques. These terms appear on each rotational axis and are due to the reaction torque produced by a change in rotational speed of the rotor propeller system. $I_{r} \dot{\omega}_{r}$ is a torque along the $\psi$ coordinate, and $I_{m} \dot{\omega}_{m} \sin \psi$ a torque along the $\varphi$ coordinate.

- Gravity effect. $G_{s}$ and $G_{c}$ result from the center of mass not being on the articulation.

- Coriolis and Centrifugal torques. Along the $\psi$ direction: Centrifugal torque $\frac{1}{2} I_{c} \dot{\varphi}^{2} \sin (2 \psi)$ and Coriolis torque $I_{m} \omega_{m} \dot{\varphi} \cos \psi$ due to the change in orientation of the kinetic momentum of the main propeller rotor system. Along the $\varphi$ direction: Coriolis torque generated by the change of inertia with respect to $\psi, I_{c} \dot{\psi} \dot{\varphi} \sin (2 \psi)$, and Coriolis torque, $I_{m} \omega_{m} \dot{\psi} \cos \psi$, due to the change in orientation of the main propeller rotor system kinetic momentum.

- Friction model along the two main axes. Presence of viscous friction $C_{\psi} \dot{\psi}$ and $C_{\varphi} \dot{\varphi}$ and Coulomb friction $C_{\varphi 0} \operatorname{sgn}(\dot{\varphi})$.

- Induced back electromotive force and motor viscous friction. The motor equations are simple first-order dynamics whose time constants depend on the parameter $F_{m}$ for the main motor and $F_{r}$ for the rear one. These parameters describe the joint effect of viscous friction and induced voltage.

\section{Flat Approximation of the Toycopter}

\subsection{Non flatness}

Flatness is the property of a system to possess certain outputs, called the flat outputs, which have the following properties: (i) The number of flat outputs is equal to the number of inputs. (ii) Given these outputs and their derivatives, all states and inputs can be expressed as a function of the flat outputs and a finite number of their derivatives $[3,4]$.

Although regular helicopters are flat [10], it has been shown in [11] that the toycopter has a defect of at most one and, using [13], that this defect is exactly one. Thus the natural outputs $\psi$ and $\varphi$ are not flat outputs, since 
the input appears already after two time differentiations leaving two states $\omega_{m}$ and $\omega_{r}$ that cannot be expressed as a combination of $\psi, \varphi$ and a finite number of their time derivatives. The presence of the input after only two differentiations is due to the reactive torque appearing when the propeller speed is varied.

One is tempted to try to control the toycopter through the use of motor inputs to impose the desired acceleration on both axes $\psi$ and $\varphi$, since these inputs appear in the expression of $\ddot{\psi}$ and $\ddot{\varphi}$. The linearized system around the equilibrium point $(\bar{x}, \bar{u})$ where $\bar{x}=$ $\left\{\bar{\psi}, \dot{\bar{\psi}}, \bar{\varphi}, \dot{\bar{\varphi}}, \bar{\omega}_{m}, \bar{\omega}_{r}\right\}$ and $\bar{u}=\left\{\bar{u}_{m}, \bar{u}_{r}\right\}$ can be expressed using the state variation $\Delta x=\bar{x}-x$. The linearised equations corresponding to (1), (2) can be solved to obtain the input variation $\Delta u_{m}=u_{m}-\bar{u}_{m}$ and $\Delta u_{r}=u_{r}-\bar{u}_{r}$ resulting from a desired acceleration of both axes $\Delta \ddot{\psi}_{c}$ and $\Delta \ddot{\varphi}_{c}$. The homogeneous part of the linearised equations corresponding to $(3),(4)$ then become

$$
\begin{aligned}
I_{m} \Delta \dot{\omega}_{m}= & 2 C_{r} \bar{\omega}_{r} \Delta \omega_{r}-2 C_{m 1} \bar{\omega}_{m} \Delta \omega_{m}- \\
& I_{m} \dot{\bar{\psi}} \frac{\cos (\bar{\psi})}{\sin (\bar{\psi})} \Delta \omega_{m} \\
I_{r} \Delta \dot{\omega}_{r}= & 2 C_{m} \bar{\omega}_{m} \Delta \omega_{m}-2 C_{r 1} \bar{\omega}_{r} \Delta \omega_{r}+ \\
& I_{m} \cos (\bar{\psi}) \dot{\bar{\varphi}} \Delta \omega_{m}
\end{aligned}
$$

Using the numerical values given in Table 9 for all possible equilibrium points $(\bar{x}, \bar{u})$, these dynamics are unstable. By redefinition of the output, it is shown in [11] that the internal dynamics can be reduced to a single dimension, and these dynamics are unstable. The main obstacle in flattening the system is due to the inertial counter torques and the particular structure of the Coriolis/Centrifugal terms. Let us emphasize that, according to [13], cancelling the internal dynamics with specific outputs (the flat outputs) is impossible for this system.

\subsection{Flat approximation}

The main problem in the control scheme of the previous section was that the main motor was used as a primary source of torque along the horizontal movement, which goes against engineering intuition. It is then natural to look for a scheme that uses the main propeller to lift the system, and the rear propeller to turn it. Examining the model equations, it would be appealing to impose the acceleration along the two main axes using the propeller speeds, since then the propellers would be used in a physically meaningful manner. For this purpose, high-gain feedback is used to impose the motor speeds.

$$
\begin{aligned}
& u_{m}=\frac{F_{m}}{K_{m}} \omega_{m}+\frac{C_{m 1}}{K_{m}} \omega_{m}\left|\omega_{m}\right|+\beta_{m}\left(\omega_{m d}-\omega_{m}\right) \\
& u_{r}=\frac{F_{r}}{K_{r}} \omega_{r}+\frac{C_{r 1}}{K_{r}} \omega_{r}\left|\omega_{r}\right|+\beta_{r}\left(\omega_{r d}-\omega_{r}\right)
\end{aligned}
$$

The following approximation using 4 states $\{\tilde{\psi}, \dot{\tilde{\psi}}, \tilde{\varphi}$, $\dot{\tilde{\varphi}}\}$ and 2 newly-defined inputs $\left\{\omega_{m d}, \omega_{r d}\right\}$ will be used to control the system. These inputs have received the notation of a propeller speed with the adjunction of the suffix $d$ since they correspond to the desired propeller speed. These inputs can also be seen as reference signals for the inner-level (high-gain) feedback loops on each engine.

$$
\begin{aligned}
I_{\psi} \ddot{\tilde{\psi}}= & C_{m} \omega_{m d}\left|\omega_{m d}\right|-C_{r 1} \omega_{r d}\left|\omega_{r d}\right|+ \\
& G_{s} \sin \tilde{\psi}+G_{c} \cos \tilde{\psi}+\frac{1}{2} I_{c} \dot{\tilde{\varphi}}^{2} \sin (2 \tilde{\psi})- \\
& C_{\psi} \dot{\tilde{\psi}}+I_{m} \omega_{m d} \dot{\tilde{\varphi}} \cos \tilde{\psi}
\end{aligned}
$$

$$
\begin{aligned}
\left(I_{\varphi}+I_{c} \sin ^{2}(\tilde{\psi})\right) \ddot{\tilde{\varphi}}= & C_{r} \omega_{r d}\left|\omega_{r d}\right| \sin \tilde{\psi}- \\
& C_{m 1} \omega_{m d}\left|\omega_{m d}\right| \sin \tilde{\psi}- \\
& I_{c} \dot{\tilde{\psi}} \dot{\tilde{\varphi}} \sin (2 \tilde{\psi})-I_{m} \omega_{m d} \dot{\tilde{\psi}} \cos \tilde{\psi}- \\
& C_{\varphi} \dot{\tilde{\varphi}}-C_{\varphi 0} \operatorname{sgn}(\dot{\tilde{\varphi}})
\end{aligned}
$$

Due to the approximation, $\{\tilde{\psi}, \dot{\tilde{\psi}}, \tilde{\varphi}, \dot{\tilde{\varphi}}\}$ differ from the system values $\{\psi, \dot{\psi}, \varphi, \dot{\varphi}\}$, and the validity of this approximation around an equilibrium point will be justified in Section 5. Moreover, the flat outputs of the reduced order model are the natural outputs $\tilde{\psi}$ and $\tilde{\varphi}$. From the outputs $\tilde{\psi}$ and $\tilde{\varphi}, \dot{\tilde{\psi}}$ and $\dot{\tilde{\varphi}}$ are directly reconstructed, and the reduced state is a trivial expression of the flat outputs and a finite number of their derivatives. Equations (7) and (8) form a system of two equations in two unknowns $\omega_{m d}$ and $\omega_{r d}$. Thus the system is flat, since solving eqns (7) and (8) gives the inputs as an expression of the output and a finite number of their derivatives. Notice that the reduced model can easily be checked to be controllable in the first approximation around any equilibrium point as long as $\psi \neq 0$ modulo $\pi$.

\section{Control Structure and Motion Planning}

\subsection{Control Structure}

The cascade control structure is depicted in Figure 2 and consists of (i) high-gain controllers (5) and (6) with gains $\beta_{m}$ and $\beta_{r}$, (ii) a linearizing controller, and (iii) outer controllers each with two repeated real poles specified by, respectively $K_{\psi}$ and $K_{\varphi}$. The linearizing controller is given by

$$
\begin{aligned}
\omega_{m d} & =\sqrt{\left|g_{\psi}\right|} \operatorname{sign}\left(g_{\psi}\right) \\
\omega_{r d} & =\sqrt{\left|g_{\varphi}\right|} \operatorname{sign}\left(g_{\varphi}\right)
\end{aligned}
$$

with $g_{\psi}$ and $g_{\varphi}$ defined as

$$
g_{\psi}=\frac{1}{C_{m}}\left[-G_{s} \sin \psi-G_{c} \cos \psi+C_{\psi} \dot{\psi}\right.
$$




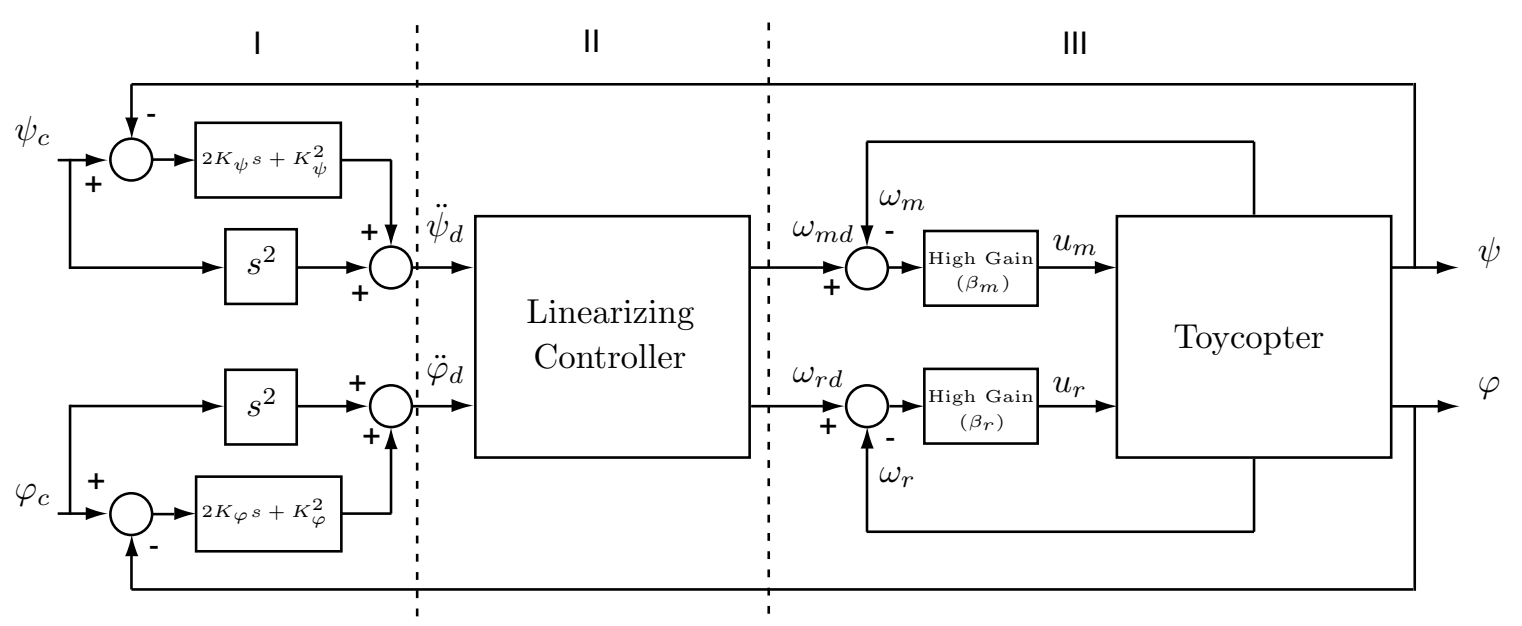

Figure 2: Block diagram of the cascade control structure. III $\approx$ flat. II + III $\approx 2 \times \frac{1}{s^{2}}$.

$$
\begin{aligned}
& -\frac{1}{2} I_{c} \sin (2 \psi) \dot{\varphi}^{2}-I_{m} \cos \psi \omega_{m} \dot{\varphi}+C_{r 1} \omega_{r}\left|\omega_{r}\right| \\
& \left.+I_{\psi} \ddot{\psi}_{d}\right] \\
g_{\varphi}= & \frac{1}{C_{r} \sin (\psi)}\left[I_{m} \omega_{m} \cos \psi \dot{\psi}+I_{c} \sin (2 \psi) \dot{\psi} \dot{\varphi}+\right. \\
& C_{m 1} \omega_{m}\left|\omega_{m}\right| \sin \psi+C_{\varphi} \dot{\varphi}+C_{\varphi_{0}} \operatorname{sign}\left(\dot{\varphi}_{d}\right)+ \\
& \left.\left(I_{\varphi}+I_{c} \sin ^{2} \psi\right) \ddot{\varphi}_{d}\right]
\end{aligned}
$$

Notice that $\ddot{\psi}_{d}$ and $\ddot{\varphi}_{d}$ correspond to the input of the linearizing controllers. The suffix $d$ is introduced to underline the fact that these inputs correspond to desired angular accelerations. These two inputs should not be confused with the angular accelerations of reference trajectories $\ddot{\psi}_{c}$ and $\ddot{\varphi}_{c}$. The outer controllers are given by $\ddot{\psi}_{d}=\ddot{\psi}_{c}+2 K_{\psi}\left(\dot{\psi}_{c}-\dot{\psi}\right)+K_{\psi}^{2}\left(\psi_{c}-\psi\right)$ and $\ddot{\varphi}_{d}=\ddot{\varphi}_{c}+2 K_{\varphi}\left(\dot{\varphi}_{c}-\dot{\varphi}\right)+K_{\varphi}^{2}\left(\varphi_{c}-\varphi\right)$.

\subsection{Motion Planning}

The control strategy will be complete once the reference trajectories $\psi_{c}$ and $\varphi_{c}$ are specified. In this paper, polynomial expressions are used to plan the motion. Since the equivalent system is a 2-2 chain of integrators, it suffices to fix a polynomial of order 5 to set the initial and terminal conditions. Since additional smoothness is desired in the trajectory planification, four extra derivatives per coordinate are added:

$$
\begin{aligned}
& \psi_{c}(t)=\psi_{c}\left(t_{0}\right)+\sum_{i=1}^{9}\left(\psi_{c}\left(t_{f}\right)-\psi_{c}\left(t_{0}\right)\right) a_{\psi i}\left(\frac{t-t_{0}}{t_{f}-t_{0}}\right)^{i} \\
& \varphi_{c}(t)=\varphi_{c}\left(t_{0}\right)+\sum_{i=1}^{9}\left(\varphi_{c}\left(t_{f}\right)-\varphi_{c}\left(t_{0}\right)\right) a_{\varphi i}\left(\frac{t-t_{0}}{t_{f}-t_{0}}\right)^{i}
\end{aligned}
$$

$t_{f}-t_{0}=T$ is the transit time needed for the reference trajectory to join the initial condition $\left(\psi_{c}\left(t_{0}\right), \varphi_{c}\left(t_{0}\right)\right)$ to the terminal conditional $\left(\varphi_{c}\left(t_{f}\right), \psi_{c}\left(t_{f}\right)\right)$. The scalars $a_{\psi i}$ and $a_{\varphi i}$ are obtained from these initial and final conditions. Typically, the initial conditions are measured on the system and the trajectory is computed according to the desired terminal position.

\section{Limitations due to approximation}

The control scheme presented in Section 4.1 uses an approximation that puts some limitation on the time response of the controlled system. The main limitation is on the gains of the outer controllers, since these gains have a direct effect on the speed of the system trajectories.

With the control scheme presented in Section 4.1, the nonlinearities are easily taken care of. However, since a cross coupling term was neglected to obtain the reduced-order model, a careful stability analysis must be carried out. For this, we will consider a simplified system corresponding to a nominal linear system around a set point given by $\psi_{c}$ for which the full analysis of the error system will be performed.

The linearized system around the operating point is given by

$$
\begin{aligned}
I_{\psi} \ddot{\psi}+I_{r} \dot{\omega}_{r} & =C_{m}^{*} \omega_{m}-C_{r 1}^{*} \omega_{r}+G \\
I_{\varphi}^{*} \ddot{\varphi}+I_{m} \dot{\omega}_{m} & =C_{r}^{*} \omega_{r}-C_{m 1}^{*} \omega_{m} \\
I_{m} \dot{\omega}_{m} & =K_{m} u_{m}-F_{m}^{*} \omega_{m} \\
I_{r} \dot{\omega}_{r} & =K_{r} u_{r}-F_{r}^{*} \omega_{r}
\end{aligned}
$$

with $C_{m}^{*}=2 C_{m}\left|\omega_{m c}\right|, C_{r}^{*}=2 C_{r}\left|\omega_{r c}\right|, C_{m 1}^{*}=$ $2 C_{m 1}\left|\omega_{m c}\right|, C_{r 1}^{*}=2 C_{r 1}\left|\omega_{r c}\right|, F_{m}^{*}=F_{m}+2 C_{m 1} \mid$ $\omega_{m c}\left|, F_{r}^{*}=F_{r}+2 C_{r 1}\right| \omega_{r c} \mid, I_{\varphi}^{*}=\frac{I_{\phi}}{\sin \psi_{c}}+I_{c} \sin \psi_{c}$ and $G=G_{s} \sin \left(\psi_{c}\right)+G_{c} \cos \left(\psi_{c}\right)$ where $\omega_{m c}$ and $\omega_{r c}$ are the steady state values of the motor speeds. Notice that most nonlinear terms vanish due the fact that the system is taken around an equilibrium where both $\dot{\varphi}$ and $\dot{\psi}$ vanish. 
Redefining appropriately $\omega_{m d}$ and $\omega_{r d}$ yields an error dynamics $\dot{e}=E e$ with error vector $e=$ $\left[\begin{array}{llllll}e_{\psi} & \dot{e}_{\psi} & e_{\varphi} & \dot{e}_{\varphi} & e_{m} & e_{r}\end{array}\right]$ and $e_{\psi}=\psi_{c}-\psi, e_{\varphi}=$ $\varphi_{c}-\varphi, e_{m}=\omega_{m c}-\omega_{m}, e_{r}=\omega_{r c}-\omega_{r}$. The following expressions are obtained after some algebraic computations:

$$
\begin{gathered}
\ddot{e}_{\psi}=-K_{\psi}^{2} e_{\psi}-2 K_{\psi} \dot{e}_{\psi}+\frac{C_{m}^{*}}{I_{\psi}} e_{m}+\frac{I_{r}}{I_{\psi}} \beta e_{r}-\frac{C_{r 1}^{*}}{I_{\psi}} e_{r} \\
\ddot{e}_{\varphi}=-K_{\varphi}^{2} e_{\varphi}-2 K_{\varphi} \dot{e}_{\varphi}+\frac{I_{m}}{I_{\varphi}^{*}} \beta e_{m}-\frac{C_{m 1}^{*}}{I_{\varphi}^{*}} e_{m}+\frac{C_{r}^{*}}{I_{\varphi}^{*}} e_{r} \\
{\left[\begin{array}{c}
\dot{e}_{m} \\
\dot{e}_{r}
\end{array}\right]=\left[\begin{array}{c}
\frac{I_{m}}{C_{m}^{*}} \psi_{c}^{(3)} \\
\frac{I_{r}}{C_{r}^{*}} \phi_{c}^{(3)}
\end{array}\right]-\beta\left[\begin{array}{c}
e_{m} \\
e_{r}
\end{array}\right]+\left[\begin{array}{cc}
C_{m}^{*} & -C_{r 1}^{*} \\
-C_{m 1}^{*} & C_{r}^{*}
\end{array}\right]^{-1}} \\
{\left[\begin{array}{c}
-2 I_{\psi} K_{\psi}^{3} e_{\psi}-3 I_{\psi} K_{\psi}^{2} \dot{e}_{\psi}+2 C_{m}^{*} K_{\psi} e_{m}+2 K_{\psi} I_{r} \beta e_{r} \\
-2 I_{\varphi}^{*} K_{\varphi}^{3} e_{\varphi}-3 I_{\varphi}^{*} K_{\varphi}^{2} \dot{e}_{\varphi}+2 K_{\varphi} I_{m} \beta e_{m}+2 C_{r}^{*} K_{\varphi} e_{r}
\end{array}\right]}
\end{gathered}
$$

The eigenvalues of the $E$ matrix are all stable as long as $K_{\psi}$ and $K_{\varphi}$ do not exceed a prescribed bound. A numerical application using the values in the appendixs and the set point $\psi_{c}=1.2, \beta_{m}=\beta_{r}=\beta=17$ shows that instability occurs when $K_{\psi}=K_{\varphi}=3.0$.

\section{Real-time experiments and comparison with simulation}

\subsection{Nominal case}

Simulation of operation is given in Figure 3. The toycopter moves in the vertical direction while creating only a small cross-coupling movement. Real-time measurements are given in Figure 4. Notice that, due to dry friction present on the horizontal axis, an offset remains. It can be shown that the controller used outperforms a standard PID controller.

\subsection{Limitation due to approximation}

It was shown in Section 5 that as long as the outer loop gains do not exceed a prescribed value, stability would be guaranteed (Figure 3) On the other hand, when these gains are increased beyond a prescribed level, instability occurs as shown in Figure 5.

\section{Conclusions}

In this paper, the case study of a helicopter-like, twodegree-of-freedom, laboratory-scale setup was considered and a control structure based on a flat approximation was proposed. A reduced order model was obtained using the propeller speeds as inputs. A control structure for the full non-flat model was then proposed. It consisted of the cascade of high-gain feedback that reduces the initial system order and a linearizing part based on this lower-order model. Since the original nonlinear model had extra couplings that were neglected in the process of obtaining the reduced-order
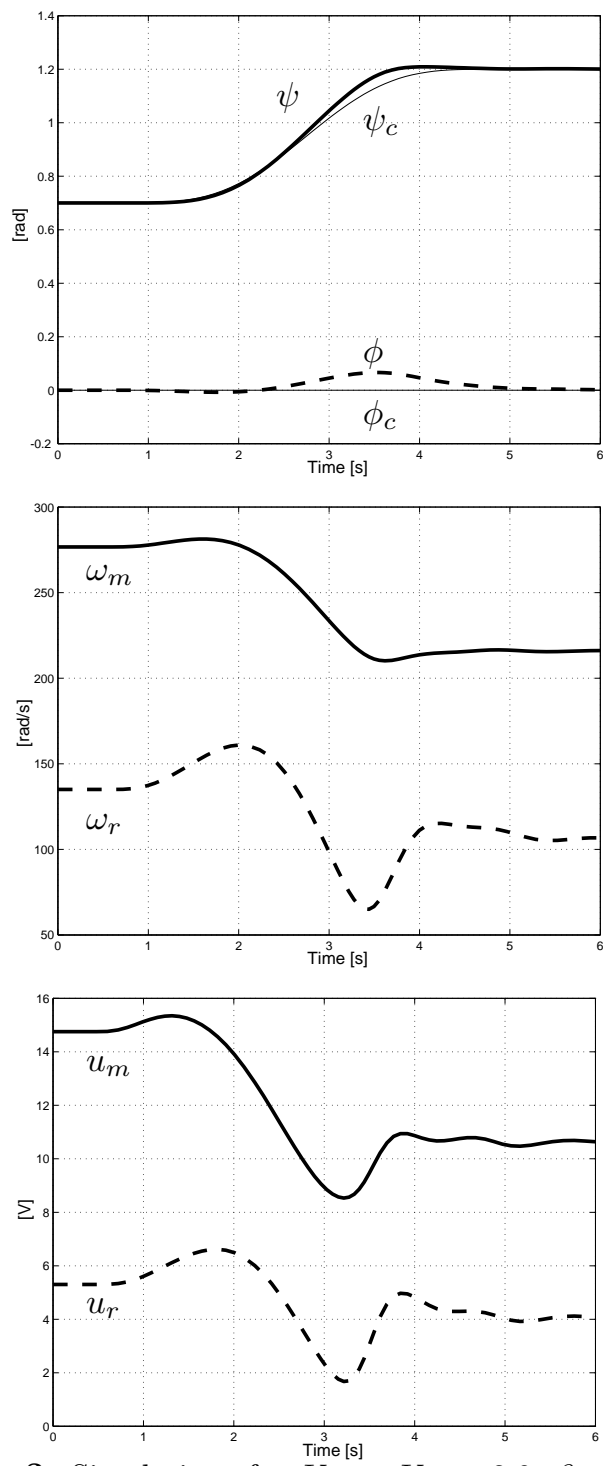

Figure 3: Simulations for $K_{\psi}=K_{\varphi}=2.0, \beta_{m}=\beta_{r}=$ 17.0. The transient time $T=4.5[\mathrm{~s}]$.

model, stability had to be assessed by taking into account the neglected term. The result is an upper bound on the gains of the outer controllers. These bounds would not exist had the original system been flat. Furthermore, the controller performed well with respect to disturbance rejection. The proposed scheme, although tuned for a particular system, seems promising as far as to encourage the usage of flat approximation for complex nonlinear dynamics.

\section{Acknowdledgments}

The authors would like to thank P. Huguenin for the real time implementation of the control scheme. 

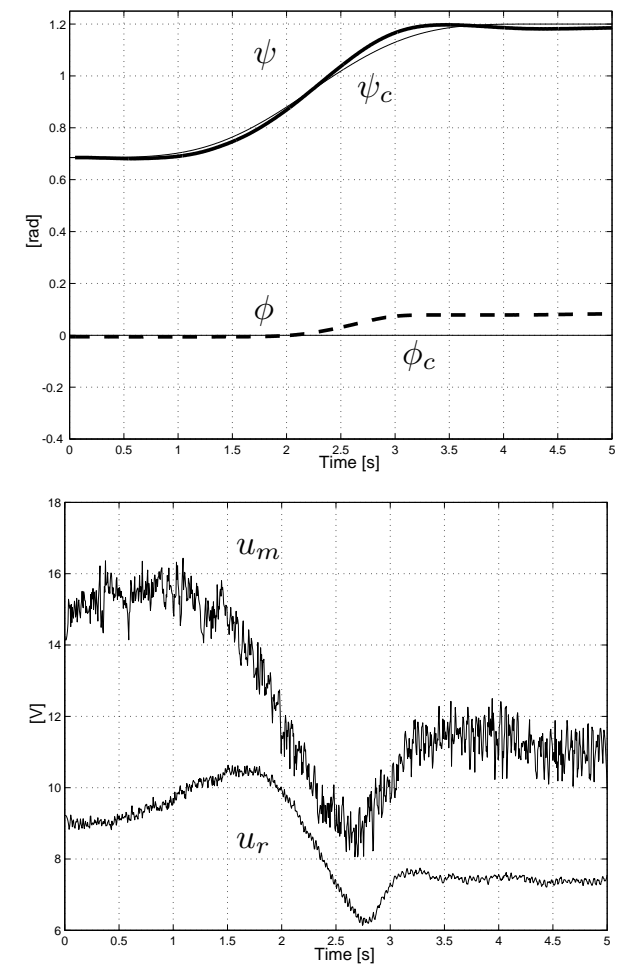

Figure 4: Real-time measurements. $K_{\psi}=K_{\varphi}=2.0$, $\beta_{m}=\beta_{r}=10$ and $T=4.5[\mathrm{~s}]$.

\begin{tabular}{|l|lr||l|lr|}
\hline$I_{\psi}$ & $40 \mathrm{e}-3$ & {$\left[\mathrm{~kg} \mathrm{~m}^{2}\right]$} & $I_{\varphi}$ & $6.7 \mathrm{e}-3$ & {$\left[\mathrm{~kg} \mathrm{~m}^{2}\right]$} \\
\hline$I_{c}$ & $31.7 \mathrm{e}-3$ & {$\left[\mathrm{~kg} \mathrm{~m}^{2}\right]$} & $C_{\varphi_{0}}$ & $24 \mathrm{e}-3$ & {$[\mathrm{Nm}]$} \\
\hline$C_{\psi}$ & $6 \mathrm{e}-3$ & {$[\mathrm{Nm} \mathrm{s} / \mathrm{rad}]$} & $C_{\varphi}$ & $2 \mathrm{e}-3$ & {$[\mathrm{Nm} \mathrm{s} / \mathrm{rad}]$} \\
\hline$C_{m}$ & $3.64 \mathrm{e}-6$ & $\left.[\mathrm{Nm} \mathrm{s})^{2} / \mathrm{rad}\right]$ & $C_{r}$ & $1.26 \mathrm{e}-6$ & {$\left[\mathrm{Nm} \mathrm{s}^{2} / \mathrm{rad}\right]$} \\
\hline$C_{m 1}$ & $3 \mathrm{e}-7$ & {$\left[\mathrm{Nm} \mathrm{s}^{2} / \mathrm{rad}\right]$} & $C_{r 1}$ & $1.6 \mathrm{e}-7$ & {$\left[\mathrm{Nm} \mathrm{s}^{2} / \mathrm{rad}\right]$} \\
\hline$I_{m}$ & $21 \mathrm{e}-5$ & {$\left[\mathrm{~kg} \mathrm{~m}^{2}\right]$} & $I_{r}$ & $54.4 \mathrm{e}-6$ & {$\left[\mathrm{~kg} \mathrm{~m}^{2}\right]$} \\
\hline$F_{m}$ & $15 \mathrm{e}-5$ & {$[\mathrm{Nm} \mathrm{s} / \mathrm{rad}]$} & $F_{r}$ & $15 \mathrm{e}-5$ & {$[\mathrm{Nm} \mathrm{s} / \mathrm{rad}]$} \\
\hline$K_{m}$ & $4.37 \mathrm{e}-3$ & {$[\mathrm{Nm} / \mathrm{V}]$} & $K_{r}$ & $4.37 \mathrm{e}-3$ & {$[\mathrm{Nm} / \mathrm{V}]$} \\
\hline$G_{s}$ & $-60 \mathrm{e}-3$ & {$[\mathrm{Nm}]$} & $G_{c}$ & -0.31 & {$[\mathrm{Nm}]$} \\
\hline
\end{tabular}

Table 1: Model parameters

\section{Appendix}

\section{References}

[1] S.A. Bortoff. Approximate state-feedback linearization using spline functions. Automatica, 33(8):1449-1458, 1997.

[2] M. Fliess. Some remarks on gain scheduling. Proc. ECC'91, Grenoble, pages 177-181, 1991.

[3] M. Fliess, J. Lévine, P. Martin, and P. Rouchon. Flatness and defect of non-linear systems: introductory theory and examples. International Journal of Control, 6:1327-1361, 1995.

[4] M. Fliess, J. Lévine, P. Martin, and P. Rouchon. A Lie-Bäcklund approach to equivalence and flatness of nonlinear systems. IEEE Trans. Automatic Control, (to appear), 1998.

[5] D. Guo and W.J. Rugh. A stability result for

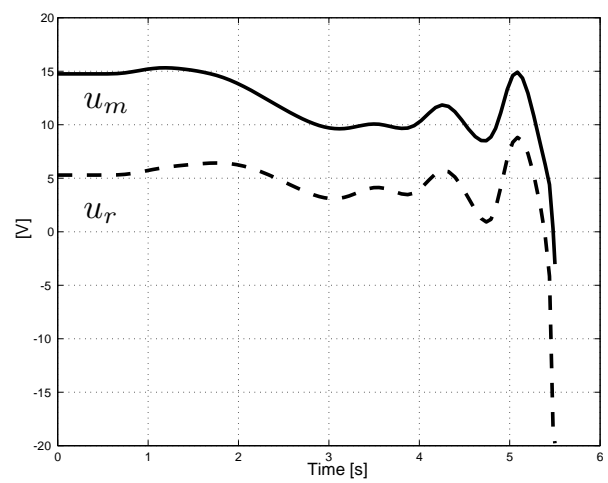

Figure 5: Instability occurs when $K_{\psi}=K_{\varphi}=3.0$.

linear parameter-varying systems. Systems $\&$ Control Letters, (24):1-5, 1995.

[6] J. Hauser. Nonlinear control via uniform system approximation. Systems \& Control Letters, (17):145154, 1991.

[7] A. Lagerberg and C. Breitholz. Study of gain scheduling control applied to an exothermic cstr. Chemical Engineering and Technology, 20(7):435-444, 1997.

[8] J. Lévine, J. Lottin, and Ponsart J.C. A nonlinear approach to the control of magnetic bearings. IEEE Control Systems Technology, 4(5):524-544, 1996.

[9] Ph. Martin. Aircraft control using flatness. Proc. CESA'96, Lille, pages 194-199, 1996.

[10] G. Meyer, R. Su, and L.R. Hunt. Application of nonlinear transformations to automatic flight control. Automatica, 20:102-107, 1984.

[11] Ph. Mullhaupt, B. Srinivasan, J. Lévine, and D. Bonvin. A toy more difficult to control than the real thing. European Control Conference, pages 431435, 1997.

[12] Y. Piguet, U. Holmberg, and R. Longchamp. Multiobjective controller design using multiple models. 2nd IFAC Symposium on Robust Control Design, pages 253-258, 1997.

[13] P. Rouchon. The toycopter is not flat. Private communication, 1997.

[14] W.J. Rugh. Analytical framework for gain scheduling. IEEE Control Systems Magazine, (11):7984, 1991.

[15] J. Shamma and M. Athans. Guaranteed properties of gain scheduled control for linear parametervarying plants. Automatica, (27):559-564, 1991. 